\title{
Evaluation of various phenotypic methods with genotypic screening for detection of methicillin- resistant Staphylococcus aureus
}

\author{
Archana Loganathan', Prasanth Manohar', Kandasamy Eniyan', Rama Jayaraj², Ramesh Nachimuthu',**
}

Abstract

Background: Staphylococcus aureus is one of the common opportunistic gram-positive pathogens which are often associated with nosocomial infections. Detection of methicillin-resistant S. aureus (MRSA) has become complicated due to the complex phenotypic and genomic pattern.

Objective: To evaluate the sensitivity and specificity pattern of various phenotypic methods used in screening mec genes harboring MRSA.

Methods: Clinical isolates of $S$. aureus were collected from diagnostic centers in Tamil Nadu. Phenotypic identification methods such as Minimal Inhibitory Concentration for oxacillin, oxacillin screen agar (OSA), oxacillin disk diffusion, and cefoxitin disk diffusion (CFD) tests were compared. The clinical isolates were classified into MRSA and methicillinsusceptible $S$. aureus (MSSA) based on the polymerase chain reaction (PCR) amplification of the mecA gene.

Result: Out of $50 \mathrm{~S}$. aureus, 21 were found to be MRSA based on the presence of the mecA gene. All 21 mecA-positive isolates were found to be resistant through minimum inhibitory concentration (MIC) and CFD test, having a sensitivity of $100 \%$ and specificity of $52 \%$ and $62 \%$, respectively. OSA and oxacillin disk tests were found to have a sensitivity of $86 \%$ and specificity of $48 \%$ and $52 \%$, respectively.

Conclusion: The combination of two phenotypic methods, CFD and oxacillin MIC, can be used for the detection of MRSA in clinical laboratories.

Keywords: cefoxitin; heterogenous population; Staphylococcus aureus

Staphylococcus aureus is one of the predominant nosocomial pathogens causing serious healthcare problems. They cause a wide range of soft tissue infections, such as endocarditis, bloodstream infection, and osteomyelitis [1]. In early 1960 s, those strains showing resistance to methicillin antibiotic were termed methicillin-resistant S. aureus (MRSA). Later this acronym is used for resistance of $S$. aureus to all $\beta$-lactam antibiotics. Genotypically, MRSA is determined by the presence of mec gene factors. At present, a new resistant variant in $S$. aureus has been developed, called borderline oxacillin-resistant $S$. aureus (BORSA), in which isolates without mec gene factor tend to show moderate resistance to $\beta$-lactam antibiotics caused by hyper $\beta$-lactamase production [2]. WHO recently listed MRSA as a highly riskiest pathogen [3]. MRSA has turned out to be a key pathogen in nosocomial infections due to its exceptional repertoire of virulence factors and its

*Correspondence to: Ramesh Nachimuthu, Department of Biomedical Sciences, School of Bioscience and Technology, Vellore Institute of Technology, Tamil Nadu, India, e-mail: ramesh.n@vit.ac.in

'Department of Biomedical Sciences, School of Bioscience and Technology, Vellore Institute of Technology, Tamil Nadu, India

2Department of Clinical Sciences, College of Health and Human Sciences, Charles Darwin University, Darwin, Northern Territory, Australia

2 Open Access. ( 2019 Loganathan et al., published by Sciendo. (cc) BY-NC-ND This work is licensed under the Creative Commons Attribution NonCommercial-NoDerivatives 4.0 License. 
ability to persist in the wide variety of antibiotic environment exhibiting heterogeneous community [4]. The incidence of MRSA in India is endemic among which the prevalence of community-associated MRSA (CA-MRSA) varies from $25 \%$ in North to $50 \%$ in southern part of India [5]. Resistance in $S$. aureus is conferred by the acquisition of a new bacterial penicillin-binding protein (PBP) called low-affinity PBP. PBP is encoded by mecA genes that confer resistance to all $\beta$-lactam antibiotics [6]. Recently, the first case of a gene conferring resistance as homology to mecA that carries novel $\mathrm{PBP}(\mathrm{mec} \mathrm{C})$ was reported. They were found to have $69 \%$ homolog to mecA gene, while the gene product had an identity of $63 \%$ [7].

The existence of a BORSA has made the detection of MRSA more complicated. BORSA isolates are characterized as small populations that exhibit an intermittent MIC ranging from 4 to $8 \mu \mathrm{g} / \mathrm{mL}$ even in the absence of mec genes [8]. These BORSA strains have made the detection method so complicated, which is predicted to exist as a result of modified acylation rate due to point mutation. But the phenotypic expression of false resistance in BORSA can vary depending on the external growth determinants such as temperature, osmolarity, and supplementations like $\mathrm{NaCl}$ and glucose [9]. The presence of mecA is highly conserved in MR staphylococci, which remains the gold standard for the detection of MRSA. But non-mecA-resistant strains are also reported. All these factors make the phenotypic MRSA identification more complicated. The phenotypic methods used for the identification of MRSA include oxacillin MIC (agar dilution/broth dilution) or E-strip, oxacillin disk diffusion, and oxacillin agar screen tests and recently identified cefoxitin disk diffusion (CFD) method. The phenotypic identification methods for MRSA in diagnostic laboratories should be simple, rapid, sensitive, and accurate. Therefore, the aim of this study is to evaluate the different phenotypic methods used for MRSA detection and to compare the sensitivity and specificity of the detection methods.

\section{Materials and methods}

\section{Staphylococcal isolates}

A total of 50 non-repetitive, Staphylococcus spp. were obtained from two diagnostic centers located in Tiruchirappalli and Chennai, Tamil Nadu from 2016 to 2017. The isolates were collected from the catheter tip, blood, pus, urine, wound swabs, bronchial wash, and sputum. Ethical approval for carrying out the study was received from Institutional Ethical Committee for Human Studies from VIT University (certificate of approval no.VIT/IECH/004/January 28, 2017). S. aureus was identified by standard microbiological techniques that involve mannitol fermentation, gram staining, and biochemical characterization such as coagulase test.

\section{Antibiotic susceptibility testing}

The antibiotic susceptibility test was performed by disk diffusion test using Mueller-Hinton agar (Hi-Media, India), following the CLSI guidelines [10]. Susceptibility test was performed using the following antibiotic disks: oxacillin $(1 \mu \mathrm{g})$, cefotaxine $(30 \mu \mathrm{g})$, cefoxitin $(30 \mu \mathrm{g})$, methicillin $(5 \mu \mathrm{g})$, penicillin $(10 \mu \mathrm{g})$, clindamycin $(2 \mu \mathrm{g})$, cefepime $(30 \mu \mathrm{g})$, ampicillin $(10 \mu \mathrm{g})$, erythromycin $(15 \mu \mathrm{g})$, gentamycin $(10 \mu \mathrm{g})$, ciprofloxacin $(5 \mu \mathrm{g})$, tetracycline $(30 \mu \mathrm{g})$, and rifampicin $(5 \mu \mathrm{g})$. ATCC 25923 was used as a quality control strain.

\section{Oxacillin agar screen test}

The oxacillin agar screen test was performed in accordance with the CLSI guidelines to detect MRSA. The test involves spot inoculation of $0.5 \mathrm{McF}$ arland standard suspension of each test isolates in Mueller-Hinton agar plates, containing 4\% of $\mathrm{NaCl}$ and $6 \mu \mathrm{g} / \mathrm{mL}$ of oxacillin, and the plate was incubated at $37^{\circ} \mathrm{C}$ for $24 \mathrm{~h}$. Those isolates that showed any visible growth was considered as MRSA and the result obtained was quality controlled by ATCC $43300[11,12]$.

\section{Minimum inhibitory concentration}

Minimum inhibitory concentration (MIC) for S. aureus was determined for oxacillin (Sigma-Aldrich, India) using microbroth dilution method as per CLSI guidelines [13]. Concentration ranging from 0.125 to $128 \mu \mathrm{g} / \mathrm{mL}$ was used for the determination of MIC. The results were interpreted as MIC $\geq 4$ as resistant and $\leq 2$ as sensitive, based on the CLSI guidelines. ATCC 25923 and ATCC 29213 were used as quality control strains [14].

\section{CFD test}

All the isolates were subjected to CFD test using a $30 \mu \mathrm{g}$ cefoxitin disk (Hi-Media, India). A bacterial suspension of $0.5 \mathrm{McF}$ arland standard was prepared; the bacterial lawn was made on Muller-Hinton agar plate; and the cefoxitin disk was placed. Plates were incubated at $37^{\circ} \mathrm{C}$ for $16-18 \mathrm{~h}$ and zone diameters were measured. ATCC 25923 and ATCC 43300 were used as negative and positive quality control strains, respectively [15]. 


\section{lodometric test for detection of $\beta$-lactamase production}

Penicillin solution (10,000 IU) of $100 \mu \mathrm{L}$ was distributed in 96-well microtiter plates. The staphylococcal isolates were suspended in wells to obtain a final concentration of $10^{9}$ cells $/ \mathrm{mL}$ (McFarland equivalent of 4 ). Ten microliters of $1 \%$ freshly prepared starch indicator was added in each well and thoroughly mixed. The titer plates were incubated at room temperature for 30-60 min. After the incubation, one drop of iodine reagent was dispensed in each well and the test was performed in triplicates. The rapid decolorization of blue color within 10 min was regarded as positive for $\beta$-lactamase production and the prolonged existence of blue color was regarded as negative. ATCC 25923 and ATCC 29213 were used as negative and positive quality control strains, respectively [16].

\section{Genomic analysis of mec genes}

Chromosomal DNA was extracted using phenol:chloroform method $[17,18]$ and it was used as a template DNA in polymerase chain reaction (PCR) for the detection of $m e c \mathrm{~A}$ and $m e c \mathrm{C}$ genes. The primers and PCR conditions used for mecA and $m e c \mathrm{C}$ gene amplification were described by García-Álvarez et al. and Khairalla et al., respectively $[19,20]$. The amplified PCR product was sequenced (Eurofins Genomics India Pvt. Ltd., Bangalore, India) and the obtained sequence results were analyzed using the NCBI-Nucleotide BLAST tool. The blast result of the obtained sequence showed $100 \%$ similarity with the existing sequence available in NCBI database. All the sequences were submitted in Genbank and accession numbers were obtained (MH459065, MH459066, MH459067, and MH459068).

\section{Statistical analysis}

All data analysis was performed using MedCalc Statistical Software version 19.1 (MedCalc Software Inc., Mariakerke, Belgium). Comparisons between four phenotypic methods with $m e c$ gene factor and cut-off value for sensitivity, specificity, positive predictive value, negative predictive value, and accuracy are provided. In the present study, validity refers to the degree to which CFD test, MIC for oxacillin test, oxacillin screen agar (OSA) test, oxacillin disk (OX) method are in agreement with the clinical diagnosis of having MRSA or not. Relative operating characteristic (ROC) curves were calculated for the determination of specificity and sensitivity of four phenotypic methods. Overall degree of validation was determined by area under the curve (AUC). Correlation coefficient between two phenotypic methods was calculated using Pearson's correlation coefficient to determine the dual phenotypic method that can be used effectively to outnumber the MRSA.

\section{Result}

A total of 50 staphylococcal isolates were used in this study, and the isolates were collected from pus $(\mathrm{n}=31)$, urine $(\mathrm{n}=2)$, sputum $(n=2)$, wound swab $(n=2)$, blood $(n=1)$, umbilical swab $(n=1)$, bronchial fluid $(n=1)$, catheter tip $(n=1)$, and some unknown $(\mathrm{n}=9)$. All the isolates were coagulase positive and confirmed to be $S$. aureus.

All the isolates studied were observed to be resistant to $\geq 3$ antibiotics, so they were categorized as multidrug resistant (MDR). The antibiotic resistance pattern was found to be highly resistant to methicillin (100\%), penicillin (98\%), ampicillin $(96 \%)$, cefotaxime $(80 \%)$ ciprofloxacin $(78 \%)$, erythromycin $(72 \%)$, cefepime $(66 \%)$, cefoxitin $(64 \%)$, oxacillin $(56 \%)$, gentamycin $(40 \%)$, tetracycline $(8 \%)$, and rifampicin $(8 \%)$ whereas clindamycin (4\%) is the least resistant antibiotic. The resistance pattern of various antibiotic disks is shown in Figure 1. The multiple antibiotic resistance (MAR) index ranged from 0.27 to 0.81 , showing that the isolates have originated from a high risk source.

In this study, four phenotypic methods that are used routinely in laboratories for the detection of MRSA were evaluated for their accuracy. The presence of mec gene (mecA/ $m e c \mathrm{C}$ ) is the major factor that confers methicillin resistance in S. aureus. Thus, the outcome of the four phenotypic methods was validated with that of mec genes, which remains the gold standard method. Test results of four phenotypic methods are provided in Table 1.

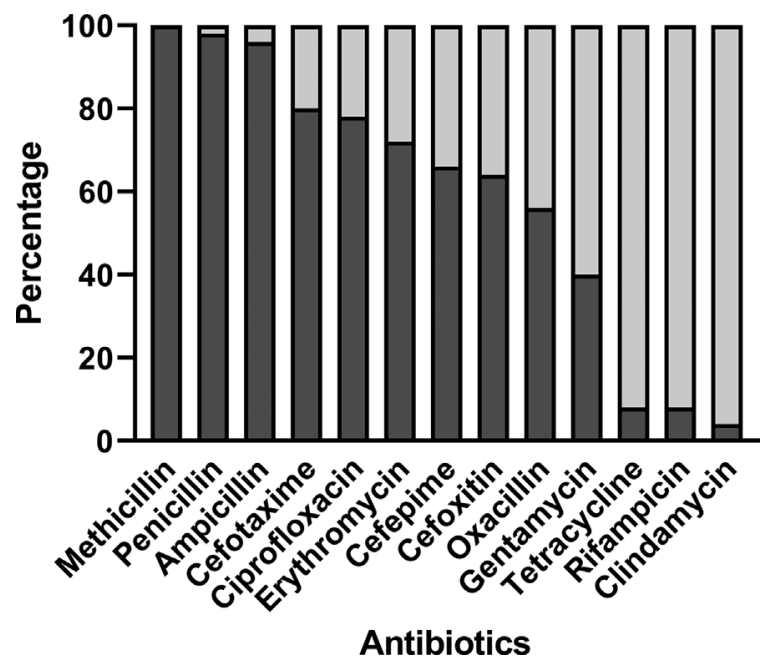

Figure 1. The percentage of resistance (dark-gray bars) and susceptibility (light-gray bars) observed for 13 antibiotics by disk diffusion test 
Table 1. Result of genotypic and phenotypic methods of 50 S. aureus

\begin{tabular}{|c|c|c|c|c|c|c|}
\hline Isolate no. & mecA gene & OSA test & Oxacillin MIC & Cefoxitin disk test & Oxacillin disk & $\beta$-Lactamase test \\
\hline SA1 & + & Growth & $128(\mathrm{R})$ & Resistant & Resistant & Positive \\
\hline SA2 & - & No growth & $1(\mathrm{~S})$ & Susceptible & Susceptible & Negative \\
\hline SA3 & + & Growth & $>128(\mathrm{R})$ & Resistant & Resistant & Positive \\
\hline SA4 & + & Growth & $64(\mathrm{R})$ & Resistant & Resistant & Positive \\
\hline SA5 & + & No growth & $8(\mathrm{R})$ & Resistant & Resistant & Positive \\
\hline SA6 & - & No growth & $8(\mathrm{R})$ & Susceptible & Resistant & Positive \\
\hline SA7 & + & Growth & $>128(\mathrm{R})$ & Resistant & Resistant & Positive \\
\hline SA8 & - & Growth & $64(\mathrm{R})$ & Resistant & Resistant & Positive \\
\hline SA9 & + & Growth & $128(\mathrm{R})$ & Resistant & Resistant & Positive \\
\hline SA10 & + & Growth & $36(R)$ & Resistant & Resistant & Positive \\
\hline SA11 & + & Growth & $>128(\mathrm{R})$ & Resistant & Resistant & Positive \\
\hline SA12 & + & No growth & $128(\mathrm{R})$ & Resistant & Resistant & Positive \\
\hline SA13 & + & Growth & $>128(\mathrm{R})$ & Resistant & Resistant & Positive \\
\hline SA14 & + & Growth & $>128(\mathrm{R})$ & Resistant & Resistant & Positive \\
\hline SA15 & + & Growth & $4(\mathrm{R})$ & Resistant & Susceptible & Positive \\
\hline SA16 & + & Growth & $8(\mathrm{R})$ & Resistant & Susceptible & Positive \\
\hline SA17 & + & Growth & $64(\mathrm{R})$ & Resistant & Resistant & Positive \\
\hline SA18 & + & Growth & $>128(\mathrm{R})$ & Resistant & Resistant & Positive \\
\hline SA19 & - & No growth & $32(\mathrm{R})$ & Resistant & Resistant & Positive \\
\hline SA20 & - & Growth & $>128(\mathrm{R})$ & Resistant & Resistant & Positive \\
\hline SA21 & + & Growth & $>128$ (R) & Resistant & Resistant & Positive \\
\hline SA22 & - & Growth & $8(\mathrm{R})$ & Susceptible & Resistant & Positive \\
\hline SA23 & - & Growth & $>128(\mathrm{R})$ & Resistant & Resistant & Positive \\
\hline SA24 & + & Growth & $>128(\mathrm{R})$ & Resistant & Resistant & Positive \\
\hline SA25 & - & No growth & $1(\mathrm{~S})$ & Susceptible & Susceptible & Positive \\
\hline SA26 & + & Growth & $>128(\mathrm{R})$ & Resistant & Resistant & Positive \\
\hline SA27 & - & No growth & $1(\mathrm{~S})$ & Susceptible & Resistant & Positive \\
\hline SA28 & - & Growth & $1(\mathrm{~S})$ & Susceptible & Susceptible & Positive \\
\hline SA29 & - & Growth & $>128(\mathrm{R})$ & Resistant & Susceptible & Positive \\
\hline SA30 & - & No growth & $64(\mathrm{R})$ & Susceptible & Susceptible & Positive \\
\hline SA31 & - & Growth & $>128(\mathrm{R})$ & Resistant & Resistant & Positive \\
\hline SA32 & - & Growth & $32(R)$ & Resistant & Resistant & Positive \\
\hline SA33 & - & Growth & $64(R)$ & Resistant & Resistant & Positive \\
\hline SA34 & - & Growth & $2(S)$ & Susceptible & Susceptible & Positive \\
\hline SA35 & - & No growth & $1(\mathrm{~S})$ & Susceptible & Susceptible & Positive \\
\hline SA36 & - & No growth & $0.5(\mathrm{~S})$ & Susceptible & Susceptible & Positive \\
\hline SA37 & + & Growth & $8(\mathrm{R})$ & Resistant & Resistant & Positive \\
\hline SA38 & - & No growth & $1(\mathrm{~S})$ & Susceptible & Susceptible & Positive \\
\hline SA39 & - & Growth & $64(\mathrm{R})$ & Resistant & Resistant & Positive \\
\hline SA40 & + & No growth & $16(\mathrm{R})$ & Resistant & Susceptible & Positive \\
\hline SA41 & - & No growth & $2(S)$ & Susceptible & Susceptible & Positive \\
\hline SA42 & - & No growth & $1(\mathrm{~S})$ & Susceptible & Susceptible & Positive \\
\hline SA43 & - & Growth & $1(\mathrm{~S})$ & Susceptible & Susceptible & Positive \\
\hline SA44 & - & No growth & $0.5(\mathrm{~S})$ & Susceptible & Susceptible & Positive \\
\hline SA45 & - & Growth & $16(\mathrm{R})$ & Resistant & Resistant & Positive \\
\hline SA46 & - & Growth & $8(\mathrm{R})$ & Resistant & Resistant & Positive \\
\hline SA47 & - & No growth & $0.5(\mathrm{~S})$ & Susceptible & Susceptible & Positive \\
\hline SA48 & + & Growth & $8(\mathrm{R})$ & Resistant & Resistant & Positive \\
\hline SA49 & - & No growth & $2(S)$ & Susceptible & Susceptible & Positive \\
\hline SA50 & - & Growth & $2(S)$ & Susceptible & Resistant & Positive \\
\hline
\end{tabular}

OSA, oxacillin screen agar; MIC, minimum inhibitory concentration; R, resistance; S, susceptible; +, positive; -, negative. 
Out of 50 isolates screened for mec gene, 21 isolates showed positive for mecA gene, which were deemed as MRSA and 29 non-mecA isolates as MSSA. None of the isolate in this study amplified for $m e c \mathrm{C}$, which shows that resistance conferred in those isolates was solely due to mecA gene. The sequenced PCR product of mecA gene was confirmed by NCBI-Nucleotide BLAST tool.

\section{Methicillin-resistance S. aureus}

Those isolates showing positive for mecA gene were regarded as MRSA. Among $21 \mathrm{mecA}$ isolates, OSA and oxacillin disk diffusion tests detected 18/3 (true positive/false negative) while MIC by oxacillin broth dilution and CFD method showed 21/0 (true positive/false negative), showing a sensitivity of $86 \%$ and $100 \%$, respectively.

\section{Borderline oxacillin resistance S. aureus}

Isolates showing non-mecA, but resistant to oxacillin with MIC range of 4-8 $\mu \mathrm{g} / \mathrm{mL}$, were considered as BORSA. Out of 50 isolates studied, 3 isolates exhibited the characteristics of BORSA. The presence of $m e c \mathrm{~A}$ was regarded as a prominent feature for MRSA, thus the presence of borderline resistance MIC in non-mecA could be due to the hyper $\beta$-lactamase production that can inhibit the $\beta$-lactam antibiotics of intermediate concentrations. Iodometric test for the detection of $\beta$-lactamase production showed hyper $\beta$-lactamase activity in $98 \%$ of the isolates. One isolate, which was non-mecA, showed a negative $\beta$-lactamase activity and it was susceptible to penicillin and oxacillin antibiotics.

\section{Methicillin-susceptible S. aureus}

Isolates with non-mecA factors were deemed MSSA. Out of 29 non-mecA isolates, OSA test detected 14/15 (true negative/ false positive), MIC by oxacillin broth dilution and oxacillin disk diffusion test detected 15/14 (true negative/false positive) while CFD method showed 18/11 (true negative/false positive), thus showing a specificity of $48 \%, 52 \%$, and $62 \%$, respectively.

In order to statistically determine the accuracy of four phenotypic studies taken into consideration, sensitivity, specificity, positive predictive value, negative predictive value, and accuracy were calculated with the aid of MedCalc Statistical Software version 19.1, which showed that CFD had a high AUC of $0.810,100 \%$ sensitivity, and $78 \%$ accuracy. The AUC and sensitivity for MIC were slightly equivalent to $\mathrm{CFD}$, whereas the AUC and sensitivity for OD and OSA were below average. ROCs for four phenotypic methods calculated are shown in Figure 2. Cut-off values showing degree of validation between experiments are calculated and represented in Table 2. From the above statistical analysis, it is obvious that CFD test and oxacillin MIC had a better accuracy to other methods studied for the detection of disease. Pearson's correlation coefficient was analyzed for the determination of correlation between phenotypic methods, which showed a high correlation for CFD+MIC with $r=0.8729$ and other methods with an average score of 0.6910 for MIC+OD, 0.6528 for OD+CFD, 0.6052 for CFD+OSA, 0.5431 for MIC+OSA, and 0.5172 for OD+OSA (Table 3).

SN, sensitivity; SP, specificity; PPV, positive predicted value; NPV, negative predicted value; AUC, area under the curve; OSA, oxacillin screen agar test; MIC, minimal inhibitory concentration; OD, oxacillin disk diffusion; CFD, cefoxitin disk diffusion.

MIC, minimal inhibitory concentration; CFD, cefoxitin disk diffusion; OD, oxacillin disk diffusion; OSA, oxacillin screen agar test.

\section{Discussion}

The infections caused by $S$. aureus are found to have high mortality and morbidity. As the prevalence of communityacquired and hospital-acquired infections caused by MRSA is increasing rapidly, it becomes necessary to detect MRSA and MSSA at their early stage using simple phenotypic methods. This study compares the four different detection methods that are routinely used for MRSA detection. Accordingly, CFD test and oxacillin MIC method were found to be more accurate than other phenotypic methods studied, as they were able to detect MRSA in all $21 \mathrm{mec}$ A-positive isolates showing 100\% sensitivity and both the above method had a better specificity of $62 \%$ and $52 \%$ when compared with other two methods. Both these methods have an advantage of less labor-intensive and they were able to detect BORSA population in infected persons. The rate of BORSA in our study was found to be $6 \%$, while previous studies by Gebhardt reported $15.8 \%$ and Khorvash et al. reported 25.5\% [21, 22]. Detection of BORSA isolates is a major limiting factor in both phenotypic and genotypic methods and improper detection of BORSA in an individual can be life-threatening for a patient. As shown in the study by Hryniewicz and Garbacz, in spite of isolates showing that non-mecA had a high MIC of $12 \mu \mathrm{g} / \mathrm{mL}$ for oxacillin and were resistant to oxacillin treatment which was further given with the combination therapy with $\beta$-lactamase inhibitor and showing that genotypic screening fails to outnumber the BORSA, it becomes necessary to determine the BORSA by dual phenotypic methods [23]. 

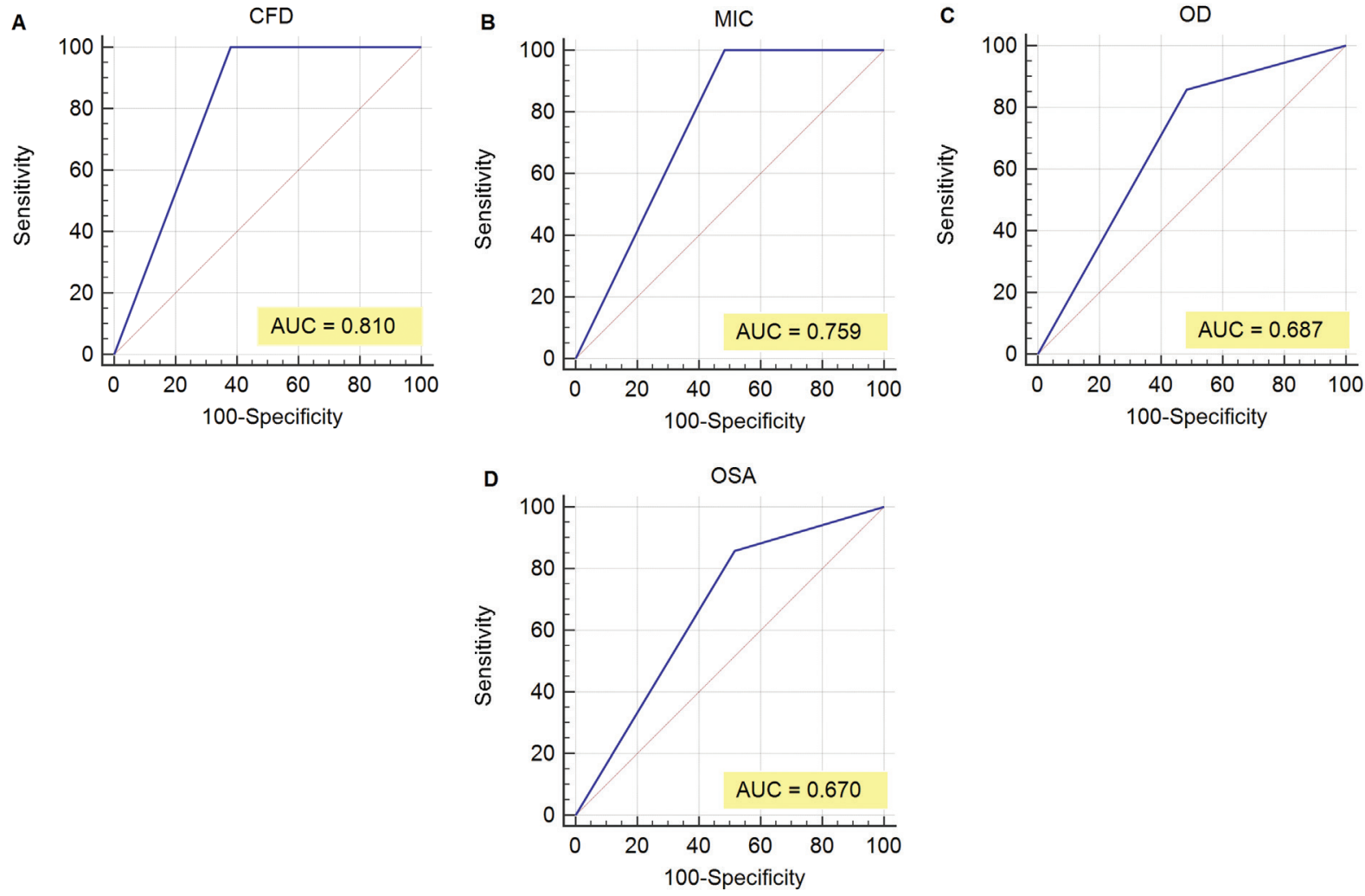

Figure 2. Receiver operating characteristics (ROCs) for (A) cefoxitin disk diffusion (CFD), (B) minimum inhibitory concentration (MIC), (C) oxacillin disk diffusion (OD), and (D) oxacillin screen agar (OSA) illustrate area under the curve (AUC) statistics

Table 2. Comparison of phenotypic methods for the detection on methicillin resistance in S. aureus

\begin{tabular}{|c|c|c|c|c|c|c|c|c|c|}
\hline \multirow{2}{*}{$\begin{array}{l}\text { Phenotypic } \\
\text { methods }\end{array}$} & \multicolumn{2}{|c|}{$\begin{array}{l}\text { No. of isolates showing mecA MRSA } \\
\qquad(n=21)\end{array}$} & \multicolumn{2}{|c|}{$\begin{array}{l}\text { No of isolates showing non-mecA } \\
\text { MSSA }(n=29)\end{array}$} & \multicolumn{5}{|c|}{ Statistical analysis (\%) } \\
\hline & $\begin{array}{l}\text { No. of true } \\
\text { positive isolates }\end{array}$ & $\begin{array}{c}\text { No. of false } \\
\text { negative isolates }\end{array}$ & $\begin{array}{c}\text { No. of true } \\
\text { negative isolates }\end{array}$ & $\begin{array}{l}\text { No. of false } \\
\text { positive isolates }\end{array}$ & SN & SP & PPV & NPV & AUC \\
\hline OSA & 18 & 3 & 14 & 15 & 86 & 48 & 54.54 & 83.35 & 64 \\
\hline MIC & 21 & 0 & 15 & 14 & 100 & 52 & 60 & 100 & 72 \\
\hline OD & 18 & 3 & 15 & 14 & 86 & 52 & 56.25 & 83.3 & 66 \\
\hline CFD & 21 & 0 & 18 & 11 & 100 & 62 & 65.6 & 100 & 78 \\
\hline
\end{tabular}

Table 3. Correlation coefficient by Pearson's correlation coefficient method for comparison of phenotypic methods

\begin{tabular}{lc}
\hline $\begin{array}{l}\text { Phenotypic methods in } \\
\text { combinations }\end{array}$ & $\begin{array}{c}\text { Pearson's rank correlation } \\
\text { coefficient }(\boldsymbol{r})\end{array}$ \\
\hline MIC+CFD & 0.8729 \\
MIC+OD & 0.6910 \\
OD+CFD & 0.6528 \\
CFD+OSA & 0.6052 \\
MIC+OSA & 0.5431 \\
OD+OSA & 0.5172 \\
\hline
\end{tabular}

Earlier Adhikari et al. had reported 100\% sensitivity in CFD and oxacillin MIC method. CFD and oxacillin MIC method showed non-mecA resistance in 15 and 18 isolates, respectively. All non-mecA isolates that showed resistance in CFD and oxacillin MIC method had a positive hyper $\beta$-lactamase activity, which could have contributed for false resistance, also the possible reason for resistance conferred by those 15 non-mecA isolates in MIC could be due to the modified expression of native PBPs, BORSA, or point mutation in resistance domain $[24,25]$. High specificity shown by CFD and oxacillin MIC was due to the presence of inducers such as cefoxitin and $\mathrm{NaCl}$ which increase the identification of 
resistance. Cefoxitin antibiotic is found to be the best inducer for PBP2a, PBP3, and PBP4 proteins [26].

The other two phenotypic methods in this study showed a low reliability, where OSA test could detect MRSA only in 18 mecA-positive $S$. aureus, and showed false positive for 15 non-mecA $S$. aureus, whereas oxacillin disk diffusion test detected MRSA in 18 mecA-positive $S$. aureus and showed a false positive for 14 non-mecA $S$. aureus. This study report that OSA test and oxacillin disk diffusion test showed a moderate sensitivity of $86 \%$ and specificity of $48 \%$ and $52 \%$, respectively, which shows the above two phenotypic methods to be moderately reliable methods for the detection of MRSA. The statistical analysis showed accuracy as follows: CFD test $>$ oxacillin MIC $>$ oxacillin disk diffusion test $>$ OSA test $(78 \%>$ $72 \%>66 \%>64 \%$ ), outlining that CFD test had a high accuracy. To prevent the erroneous outcome in the detection of MRSA, due to various external and internal factors it requires dual phenotypic analysis. To statistically determine the best dual phenotypic analysis, Pearson's correlation coefficient was calculated, which showed that CFD and oxacillin MIC had a better outcome with $r=0.8729$.

A recent study by Basset et al. showed that the presence of $m e c \mathrm{C}$ gene is another factor that confers methicillin resistance in $S$. aureus, which has a $60 \%$ of homology to mecA gene. None of the isolates in our study amplified for mec $\mathrm{C}$ gene, which states that the resistance confers in our isolates was not due to mec $\mathrm{C}$ gene [27]. Thus, disparity obtained in our study for those non-mecA-resistant $S$. aureus might be due to hyperproduction of $\beta$-lactamase; this is high in comparison with findings by Adhikari et al. [28].

In the present study, $98 \%$ of the isolates were $\beta$-lactamase producers estimated by both penicillin disk diffusion test and iodometric method. Globally, $\beta$-lactamase rate for staphylococci lies from $56 \%$ to $93 \%$ [28]. Penicillin can be preferred as a treatment for MSSA infections which are penicillinase nonproducers [29]. In our study, one MSSA showed susceptible to penicillin antibiotic and negative for $\beta$-lactamase production. All the isolates showed resistance to methicillin in disk diffusion test, including one penicillinase nonproducer. The rate of MRSA in our study was found to be $42 \%$, which is high in comparison with a report given by Kogan et al. This illustrates that rate of the MRSA infection is escalating day by day [30, 31] and simple phenotypic MRSA detection methods such as CFD and oxacillin MIC can be preferred in routine clinical laboratories. In spite of various phenotypic and genotypic methods available for detecting methicillin resistance in S. aureus, the most predominating methods used in major laboratories were found to be disk diffusion and VITEK systems, which cannot outnumber the presence of BORSA and heterogenic populations. Even though the detection of mecA gene remains the gold standard method, the inability of genomic screening to detect the BORSA and hetero-resistant variant remains the drawback. Of all phenotypic methods studied earlier, the high accuracy was found to be shown by CFD test, which is easy and labor-intensive, whereas OSA test had an advantage in effective detection of BORSA populations which can be used during the preparation of treatment to prevent the improper therapy, thereby preventing the risk of antibiotic resistance.

\section{Conclusion}

This study aims to bring to notice that there is a high change that few MRSA strains are undetected due to the lack of improper expression of resistant proteins that require inducers. Of all phenotypic methods studied earlier, the high accuracy was found to be shown by CFD test which is easy and labor-intensive, whereas genotypic screening and oxacillin MIC methods had an advantage in effective detection of BORSA populations. Even though the detection of BORSA was unpredictable, infection due to BORSA in an individual can be treated effectively by $\beta$-lactamase inhibitors. Thus this study concludes that screening of MRSA has become complicated in spite of various phenotypic methods available, and the existence of non-mecA resistance makes the phenotypic methods unreliable resulting in treatment failure and rise in antibiotic resistance. Despite the presence of PCR for the simple and early location of the resistance gene in an organism, many laboratories still rely on phenotypic output for treatment option as in MIC and other fundamental tests. Thus, there is a need for dual phenotypic analysis and genotypic screening and also for improvising the phenotypic methods with external factors that can induce the existence of resistome system which will help in treatment regimen and prevention of rising antibiotic resistance.

Data availability. The NCBI accession numbers for mecA genes were MH459065, MH459066, MH459067, and MH459068.

Author contributions. All the authors contributed substantially to the conception and design of this study. AL, PM, and $\mathrm{RN}$ contributed substantially to the acquisition and interpretation of the data. AL, PM, and RN drafted the manuscript. KE and RJ contributed substantially to its critical revision. All the authors approved the final version submitted for publication and take responsibility for the statements made in the published article.

Acknowledgments. The authors would like to thank Vellore Institute of Technology for funding support in the form of a VIT Seed Grant. 
Conflict of interest statement. The authors have completed and submitted the International Committee of Medical Journal Editors Uniform Disclosure Form for potential conflicts of interest. None of the authors disclose any conflict of interest.

\section{References}

[1] Davis KA, Stewart JJ, Crouch HK, Florez CE, Hospenthal DR. Methicillin-resistant Staphylococcus aureus (MRSA) nares colonization at hospital admission and its effect on subsequent MRSA infection. Clin Infect Dis. 2004; 39:776-82.

[2] Enright MC, Robinson DA, Randle G, Feil EJ, Grundmann H, Spratt BG. The evolutionary history of methicillin-resistant Staphylococcus aureus (MRSA). Proc Natl Acad Sci U S A. 2002; 99:7687-92.

[3] World Health Organization. Global priority list of antibioticresistant bacteria to guide research, discovery, and development of new antibiotics. [online] 2017. [cited 2018 Jan 11]. Available from: http://www.who.int/mediacentre/news/releases/2017/bacteriaantibiotics-needed/en/

[4] Popovich KJ, Weinstein RA, Hota B. Are community-associated methicillin-resistant Staphylococcus aureus (MRSA) strains replacing traditional nosocomial MRSA strains? Clin Infect Dis. 2008; 46:787-94.

[5] Indian Network for Surveillance of Antimicrobial Resistance (INSAR) group, India. Methicillin resistant Staphylococcus aureus (MRSA) in India: prevalence and susceptibility pattern. Indian Network for Surveillance of Antimicrobial Resistance [INSAR] group, India. Indian J Med Res. 2013; 137:363-9.

[6] Basanisi MG, La Bella G, Nobili G, Franconieri I, La Salandra G. Genotyping of methicillin-resistant Staphylococcus aureus (MRSA) isolated from milk and dairy products in south Italy. Food Microbiol. 2017; 62:141-6.

[7] Kriegeskorte A, Ballhausen B, Idelevich EA, Köck R, Friedrich AW, Karch H, et al. Human MRSA isolates with novel genetic homolog, Germany. Emerging Infect Dis. 2012; 18:1016.

[8] Argudín MA, Roisin S, Nienhaus L, Dodémont M, de Mendonça R, Nonhoff C, et al. Genetic diversity among S. aureus isolates showing oxacillin and/or cefoxitin resistance not linked to the presence of mec genes. Antimicrob Agents Chemother. 2018: AAC-00091.

[9] Resende CA, Figueiredo AM. Discrimination of methicillinresistant Staphylococcus aureus from borderline-resistant and susceptible isolates by different methods. J Med Microbiol. 1997; 46:145-9.

[10] Clinical and Laboratory Standards Institute. Performance standards for antimicrobial susceptibility testing. [online] 2017. [cited 2018 Jan 11]. Available from: https://clsi.org/media/1469/m100s27_sample.pdf

[11] National Committee for Clinical Laboratory Standards. Methods for dilution antimicrobial susceptibility tests for bacteria that grow aerobically. 4th ed. vol. 17, no. 2. Wayne, PA: National Committee for Clinical Laboratory Standards document M7-A4; 1997.

[12] Shrestha B, Pokhrel BM, Mohapatra TM. Phenotypic characterization of nosocomial isolates of Staphylococcus aureus with reference to MRSA. J Infect Dev Countries. 2009; 3:554-60.

[13] National Committee for Clinical Laboratory Standards. Methods for dilution antimicrobial susceptibility tests for bacteria that grow aerobically. Wayne, PA: National Committee for Clinical Laboratory Standards document M07-A9; 2012

[14] National Committee for Clinical Laboratory Standards. Performance standards for antimicrobial susceptibility testing: twenty third informational supplement edition. Wayne, PA: National Committee for Clinical Laboratory Standards document M100-S23; 2013.

[15] Swenson JM, Spargo J, Tenover FC, Ferraro MJ. Optimal inoculation methods and quality control for the NCCLS oxacillin agar screen test for detection of oxacillin resistance in Staphylococcus aureus. J Clin Microbiol. 2001; 39:3781-4.

[16] Bidya S, Suman RS. Comparative study of three $\beta$ lactamase test methods in Staphylococcus aureus isolated from two Nepalese hospitals. Open J Clin Diagn. 2014; 4:47.

[17] Sambrook JF, Russell DW, editors. Molecular cloning: a laboratory manual, vol. 1, 2, 3. New York: Cold Spring Harbor Laboratory Press; 2001.

[18] Prasad S, Nayak N, Satpathy G, Nag HL, Venkatesh P, Ramakrishnan S, et al. Molecular and phenotypic characterization of Staphylococcus epidermidis in implant related infections. Indian J Med Res. 2012; 136:483.

[19] García-Álvarez L, Holden MT, Lindsay H, Webb CR, Brown DF, Curran MD, et al. Methicillin-resistant Staphylococcus aureus with a novel mecA homologue in human and bovine populations in the UK and Denmark: a descriptive study. Lancet Infect Dis. 2011; 11:595-603.

[20] Khairalla AS, Wasfi R, Ashour HM. Carriage frequency, phenotypic, and genotypic characteristics of methicillin-resistant Staphylococcus aureus isolated from dental health-care personnel, patients, and environment. Sci Rep. 2017; 7:7390.

[21] Gebhardt DO. MRSA in the Netherlands: preventive measure raises a moral issue. J Med Ethics. 2003; 29:212.

[22] Khorvash F, Mostafavizadeh K, Mobasherizadeh S. Frequency of mecA gene and borderline oxacillin resistant Staphylococcus aureus in nosocomial acquired methicillin resistance Staphylococcus aureus infections. Pak J Biol Sci. 2008; 11:1282-5.

[23] Hryniewicz MM, Garbacz K. Borderline oxacillin-resistant Staphylococcus aureus (BORSA) - a more common problem than expected? J Med Microbiol. 2017; 66:1367-73.

[24] Huang YT, Liao CH, Chen SY, Hsu HS, Teng LJ, Hsueh PR. Emergence of multidrug-resistant sequence type 45 strains among mecA-positive borderline oxacillin-resistant Staphylococcus aureus causing bacteraemia in a medical centre in Taiwan. Int J Antimicrob Agents. 2018; 52:70-5.

[25] Sasirekha B, Usha MS, Amruta AJ, Ankit S, Brinda N, Divya R. Evaluation and comparison of different phenotypic tests to detect methicillin resistant Staphylococcus aureus and their biofilm production. Int J Pharm Tech Res. 2012; 4:532-41.

[26] Bonjean M, Hodille E, Dumitrescu O, Dupieux C, Mongo CN, Allam C, et al. Disk diffusion testing for detection of methicillinresistant Staphylococci: does moxalactam improve upon cefoxitin? J Clin Microbiol. 2016; 54:2905-9.

[27] Basset P, Prod'hom G, Senn L, Greub G, Blanc DS. Very low prevalence of methicillin-resistant Staphylococcus aureus carrying the mecC gene in western Switzerland. J Hosp Infect. 2013; 83:257-9.

[28] Adhikari R, Pant ND, Neupane S, Neupane M, Bhattarai R, Bhatta S, et al. Detection of methicillin resistant Staphylococcus aureus and 
determination of minimum inhibitory concentration of vancomycin for Staphylococcus aureus isolated from pus/wound swab samples of the patients attending a tertiary care Hospital in Kathmandu, Nepal. Canadian Can J Infect Dis Med Microbiol. 2017; 2017:2191532.

[29] Kaase M, Lenga S, Friedrich S, Szabados F, Sakinc T, Kleine B, Gatermann SG. Comparison of phenotypic methods for penicillinase detection in Staphylococcus aureus. Clin Microbiol Infect. 2008; 14:614-6.
[30] Kogan S, Zeng Q, Ash N, Greenes RA. Problems and challenges in patient information retrieval: a descriptive study. Proc AMIA Symp. 2001: 329-33.

[31] San Sit P, Teh CS, Idris N, Sam IC, Omar SF, Sulaiman H, et al. Prevalence of methicillin-resistant Staphylococcus aureus (MRSA) infection and the molecular characteristics of MRSA bacteraemia over a two-year period in a tertiary teaching hospital in Malaysia. BMC Infect Dis. 2017; 17:274. 